\title{
Argumente für eine neue Erweiterungsstrategie - die Diskussion über die Aufnahmefähigkeit der EU
}

\author{
Kai-Olaf Lang und Daniela Schwarzer*
}

Das Verhältnis von Vertiefung und Erweiterung in der Europäischen Union ist seit den Jahren 2004/2005 von zwei Paradoxen geprägt. Das erste: Seit der Ablehnung des EU-Verfassungsvertrags in den Referenden in Frankreich und den Niederlanden im Mai und Juni 2005 sind markante Fortschritte der Erweiterungspolitik gelungen. ${ }^{1}$ Dies geschah, obwohl sich in den Referendumsdiskussionen eine Ablehnung vorangegangener und künftiger Erweiterungsschritte zeigt - und obwohl eine sogenannte ,Erweiterungsmüdigkeit ' in der EU Einzug gehalten hat, die sich neben einer wachsenden Aversion der öffentlichen Meinung gegenüber weit gespannten Beitrittsversprechungen in einer zunehmend kritischeren Haltung des Europäischen Parlaments manifestiert. Das zweite Paradox ist, dass zwar schon bei der politischen Bewertung der Ergebnisse des Nizza-Gipfels von 2000 hervorgehoben wurde, dass die EU auf dieser Grundlage nicht auf mehr als 27 Staaten anwachsen könne. Doch statt das Thema institutionelle Reformen - parallel zur ungebremst fortgeschriebenen Erweiterungspolitik - aktiv weiterzuverfolgen, zogen die EU-Staaten sich nach den gescheiterten Referenden zunächst in sichtbarer Hilflosigkeit in eine ,Denkpause‘ zurück. In längst nicht allen Hauptstädten ist seither in Bezug auf die weitere Vertiefung der Union, und noch weniger in Bezug auf die laufenden Erweiterungsprozesse ein deutlicher politischer Führungswille erkennbar. Diese Zusammenhänge und Widersprüche haben dazu geführt, dass die Frage der Erweiterungsfähigkeit seit dem Jahr 2005 verstärkt in den Vordergrund der Diskussion gelangt ist und sich politische Stimmen mehren, die weitere Erweiterungsschritte - auch bezüglich der Türkei, mit der Verhandlungen am 3. Oktober 2005 begonnen haben in Frage stellen. Dies legt die Frage nahe, ob nicht - trotz der vordergründigen Geschwindigkeit der Erweiterungspolitik - ein Paradigmenwechsel in der Erweiterungslogik der Union stattfindet, der die innere Solidität und Aufnahmefähigkeit der Gemeinschaft vor die mit der Aufnahme neuer Mitgliedsstaaten verbundenen Ziele setzt.

Dieser Artikel argumentiert, dass dies bislang nicht der Fall ist. In der neuen Erweiterungsstrategie der EU-Kommission, die der Europäische Rat im Dezember 2006 unverändert angenommen hat, ist die interne Stabilisierung und Vertiefung zwar als Begleitpolitik weiter etabliert. Die Politik hat jedoch bislang die Frage unbeantwortet gelassen, wie eine konstruktive Neubalancierung beider Kalküle unter dem Primat der internen Handlungsfähigkeit ohne Verzicht auf die Transformationskapazitäten der Union und unter Berücksichtigung möglicher Blockaden des Beitrittsgeschehens durch Vetospieler bei der Ratifizierung der Beitrittsverträge zustandegebracht werden kann. Um dieses Problem zu lösen, sollte die EU eine Sequenzierung von Vertiefung und Erweiterung anstreben. Weitere Erweiterungsschritte sollten mit transparenten Kriterien zur EU-Aufnahmefähigkeit und einer stärkeren

* Kai-Olaf Lang, wissenschaftlicher Mitarbeiter, Forschungsgruppe EU-Integration der Stiftung Wissenschaft und Politik, Berlin.

Dr. Daniela Schwarzer, wissenschaftliche Mitarbeiterin, Forschungsgruppe EU-Integration der Stiftung Wissenschaft und Politik, Berlin.

1 Am 3. Oktober 2005 begannen die Beitrittsverhandlungen mit der Türkei und Kroatien. Im Dezember 2005 hat die Europäische Kommission Mazedonien den Status eines Beitrittskandidaten verliehen, die Verhandlungen haben noch nicht begonnen. 
Einbindung von nationalen Parlamenten begleitet werden. Dabei sollte nicht der Versuch unternommen werden, eine absolute Aufnahmefähigkeit der EU zu bestimmen, sondern diese mit Blick auf konkrete Kandidatenländer zu definieren und politisch zu kommunizieren. Auch dies ist allerdings angesichts möglicher Vetospieler auf nationaler und europäischer Ebene keine Garantie für den Erfolg laufender und künftiger Erweiterungsvorhaben, kristallisiert sich aber zunehmend als notwendige Voraussetzung heraus.

\section{Kein Paradigmenwechsel in der Erweiterungspolitik}

Die Diskussion um die Aufnahmefähigkeit der Europäischen Union ist nicht neu. Bereits 1993, als die Weichen für den Beitritt der mittel- und osteuropäischen Länder zur EU gestellt wurden, bezeichnete der Europäische Rat in Kopenhagen die Aufnahmefähigkeit der EU als ,wichtigen Gesichtspunkt“, da die Beibehaltung der Integrationsdynamik im Interesse der Mitgliedstaaten und der Kandidatenländer läge. ${ }^{2}$ Zudem legte die EU-Kommission bei jeder Erweiterungsrunde eine Analyse über die Auswirkungen des Beitritts neuer Mitglieder vor, etwa auf die Finanzierung der EU-Politiken oder auf das Funktionieren der rotierenden Ratspräsidentschaften. Der ehemalige deutsche Außenminister Joschka Fischer, Befürworter eines Beitritts der Türkei, unterstrich in seiner Humboldt-Rede zur Zukunft der EU im Mai $2000^{3}$ gleichermaßen die Bedeutung der Aufnahmefähigkeit der Gemeinschaft. In den Strategiedokumenten zur EU-Erweiterung der Europäischen Kommission hat die Aufnahmefähigkeit als das erste der drei bekannten K-Wörter (Konsolidierung, Konditionalität, Kommunikation) ihren festen Platz.

Seit gut eineinhalb Jahren erlebt dieses Konzept, das auch anlässlich der Aufnahme der Beitrittsgespräche mit der Türkei und einiger Balkanstaaten wieder als Grundvoraussetzung betont wurde, eine neue politische Konjunktur. Insbesondere Frankreich und Österreich haben sich dafür ausgesprochen, eindeutige Absorptionskriterien zu definieren und künftige Erweiterungsschritte von der Aufnahmefähigkeit der EU abhängig zu machen. Das Europäische Parlament hat sich in mehreren Berichten mit dem Thema befasst und forderte die EUKommission in einer Resolution dazu auf, eine umfassende Erklärung zur Aufnahmekapazität der Union zu erstellen. ${ }^{4}$

\section{Der Sonderbericht der EU-Kommission}

Am 8. November 2006 stellte die EU-Kommission ihre Mitteilung zur Erweiterungsstrategie vor. ${ }^{5}$ Diese war verbunden mit einem ,Sonderbericht über die Fähigkeiten der EU zur Integration neuer Mitglieder“, der nicht weniger beinhalten will als ,ein Konzept, das sicherstellen soll, dass die EU ihre Funktionsfähigkeit behält, während gleichzeitig eine Erweiterungsagenda umgesetzt wird, die den Herausforderungen der kommenden Jahre gerecht wird." Bereits damit wurde die eigentliche Stoßrichtung deutlich: Der alte Widerstreit zwischen Vertiefung und Erweiterung soll nicht dadurch aufgelöst werden, dass die Union expansionsreif wird und danach weitere Beitritte erfolgen. Vielmehr geht es darum, beide Prozesse parallel voranzubringen und zu optimieren.

2 Europäischer Rat (Kopenhagen): Schlussfolgerungen des Vorsitzes, 21./22.06.1993, S. 14.

3 Vgl. Joschka Fischer: Vom Staatenbund zur Föderation - Gedanken über die Finalität der europäischen Integration, in: integration 3/2000, S. 149-156.

4 Europäisches Parlament: Bericht über das Strategiepapier 2005 der Kommission zur Erweiterung, 03.02.2006, A6-0025/2006.

5 Europäische Kommission: Erweiterungsstrategie und wichtigste Herausforderungen für den Zeitraum 20062007 mit Sonderbericht über die Fähigkeit der EU zur Integration neuer Mitglieder, KOM(2006) 649 endgültig, 08.11.2006. 
Im Sonderbericht der Kommission wird das Thema Integrationsfähigkeit definiert als „Frage, ob die EU zu einem bestimmten Zeitpunkt oder in einem bestimmten Zeitraum neue Mitglieder aufnehmen kann, ohne ihre in den Verträgen begründeten politischen Ziele aufs Spiel zu setzen“. Das Integrationsvermögen der Union wird hierbei entlang dreier Dimensionen thematisiert:

- die Union soll die „Dynamik der europäischen Integration“ beibehalten:

- Kandidatenländer sollen die an sie gestellten, ,,strengen“ Bedingungen erfüllen, und

- die unionsinterne Kommunikation über Beitritte und deren Folgen soll verbessert werden.

Im Zusammenhang mit dem ersten Bereich werden drei Einzelaspekte hervorgehoben: Institutionen, EU-Politiken sowie der Haushalt. Mit Blick auf das Funktionieren der Institutionen konstatiert der Sonderbericht, dass der Vertrag von Nizza - abgesehen von der relativen Reduktion der Kommissionsgröße - keine Regelungen für eine Union mit mehr als 27 Mitgliedern enthält. Hieraus ergibt sich für die Union die Notwendigkeit, vor künftigen Erweiterungsschritten über „Inhalt und Umfang institutioneller Reformen“ entscheiden zu müssen. Die Kommission will in Zukunft bereits als ummittelbare Reaktion auf neue Beitrittsanträge die möglichen Konsequenzen entsprechender Erweiterungsschritte für die Institutionen bewerten. Gleichzeitig wird angemahnt, dass ein neues institutionelles Arrangement ,bis zu dem Zeitpunkt gefunden werden [sollte], zu dem der nächste neue Mitgliedstaat voraussichtlich die Beitrittsreife erreichen wird.“

Auch hinsichtlich der EU-Politiken ist eine prospektive und kontinuierliche Bewertung von Beitrittsfolgen geplant. Maxime soll hierbei sein, dass die Fortentwicklung und Realisierung gemeinsamer Politiken nicht beeinträchtigt werden darf. Die ,reibungslose Integration' künftiger Beitrittsländer und eine konsequente Umsetzung von EU-Politiken nach neuen Erweiterungsrunden sollen etwa durch Übergangsfristen, Ausnahme- und Sonderregelungen sowie unbefristete Schutzklauseln gewährleistet werden. Besonderes Augenmerk gilt sensitiven Politikfeldern wie Freizügigkeit, Grenzverwaltung, Landwirtschaft, Kohäsionspolitik und Verkehr, aber auch Außen- und Sicherheitspolitik sowie Energie. Die Vorabeinschätzung von Beitrittsfolgen soll es der Union ermöglichen, frühzeitig Übergangsfristen oder andere Sonderregelungen geltend zu machen.

Was die finanziellen Implikationen angeht, so soll ebenfalls bereits bei der Begutachtung künftiger Beitrittsbegehren durch die Kommission eine Schätzung der Folgewirkungen der Aufnahme des betreffenden Landes für den EU-Haushalt erfolgen. Maßgabe ist auch hier die Aufrechterhaltung eines nachhaltigen Finanzierungssystems der Union.

Die zweite Dimension der Integrationsfähigkeit stellt die ,Beitrittsfitness“ der Kandidatenländer dar. Diesbezügliches Kernelement des Sonderberichts ist das Postulat nach mehr und strikterer Konditionalität. Der Qualitätssicherung und -verbesserung sollen eine stärkere Konzentration von Hilfsmitteln auf Reformprioritäten, ein permanentes und detailliertes Monitoring von Reformfortschritten und die Verifizierung der Implementation von Anpassungsleistungen dienen. Durch die Festlegung und Überprüfung von Benchmarks unter anderem bei Eröffnung und Abschluss von Verhandlungskapiteln soll das Tempo des Beitrittsprozesses an die tatsächliche Umsetzung von Reformen gekoppelt werden. Seitens der EU soll daher kein konkreter zeitlicher Horizont in Form eines Zieldatums für den Beitritt festgelegt werden.

Als drittes Element der Integrationsfähigkeit sollen Erweiterungsrunden mit voller Bejahung der Bürger der Union vollzogen werden. Hierfür sollen einerseits ein „strenger“ Beitrittsprozess und „uneingeschränkte Konditionalität“ sorgen. Andererseits will man den Bürgern die Erweiterung besser als bislang „erklären“. Mehr Kommunikation, größere 
Transparenz und eine unnachsichtige Verhandlungsführung sollen das gesamte Erweiterungsgeschehen auf ,eine festere demokratische Grundlage“" stellen. ${ }^{6}$

Aus den Reihen des Europäischen Parlaments kam heftige Kritik an der durch die Kommission vorgenommenen Beurteilung. In einem vom Ausschuss für Auswärtige Angelegenheiten lancierten (und später vom Parlament angenommenen) Bericht (Brok-Bericht) wird unter anderem „,bedauert“, dass „die Kommission keine ausreichend tief greifende Analyse der Fragen vorlegt, die geklärt werden müssen, bevor die Union mit künftigen Erweiterungen fortfahren kann“. Der Abschnitt über die Integrationsfähigkeit der Union sei gar ,eine nicht zufrieden stellende Antwort auf die Aufforderung des Parlaments, ,einen Bericht vorzulegen, in dem sie die

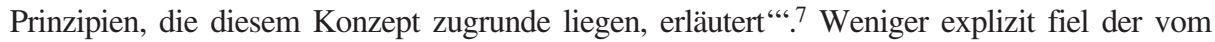
Ausschuss für Verfassungsfragen vorgelegte Stubb-Bericht aus. Dieser forderte eine umfassende institutionelle Reform als Voraussetzung für die Aufnahme weiterer Staaten und konstatierte klar, dass der Vertrag von Nizza keine ausreichende Basis für künftige Erweiterungen darstelle. $^{8}$

\section{Fehlende Antworten beim Dezemberrat}

Ende 2006 befasste sich der Europäische Rat auf Grundlage der Erweiterungsstrategie der Kommission mit der Integrationsfähigkeit der Europäischen Union. Die Formulierungen in den Ratsschlussfolgerungen ${ }^{9}$ zeugen davon, dass unter den Mitgliedstaaten gegenwärtig keine Einigkeit darüber besteht, eine Wende in der Erweiterungspolitik herbeizuführen, die die Sicherung der Integrationsdynamik über die Expansion der Union stellen würde. Die implizite Philosophie lautet: Synchronisierung, nicht Sequenzierung beider Vorhaben. Anstelle von harten, an der Aufnahme- und Funktionsfähigkeit der EU orientierten Kriterien wird eine ,Erweiterungsbegleitpolitik' gewählt: Stringenter geführte Beitrittsverhandlungen und Folgenabschätzungen für sensitive Politikbereiche sollen Befürchtungen der Bürger zerstreuen und eine reibungslose Aufnahme künftiger Mitglieder sicherstellen.

Mit der Annahme des Kommissionsberichts hat der Europäische Rat keine Abkehr vom zuletzt praktizierten Primat der Erweiterung vor der Vertiefung vollzogen. Insofern handelt es sich um eine unzureichende Antwort auf die Probleme, denen sich der Erweiterungsprozess der Europäischen Union im Grunde schon seit der Erweiterung um die mittel- und osteuropäischen Länder gegenüber sieht.

Dazu gehört erstens die bewiesene Schwäche der Doppelstrategie gleichzeitiger Erweiterung und Reform. Bereits im Kontext der Erweiterung um die zehn plus zwei Staaten in den Jahren 2004 und 2007 scheiterte der Versuch, die als nötig erachteten Vertiefungsschritte unter dem Druck der größeren Mitgliederzahl der EU umzusetzen. Trotz der bereits 1993 betonten Notwendigkeit, die Absorptionskraft der EU zu garantieren, erfolgte kein Junktim zwischen Vertiefung und Reform, da die „Erweiterung möglicherweise unterbrochen oder gar abgebrochen hätte werden müssen - mit nicht absehbaren politischen Verwerfungen “. ${ }^{10}$

6 Europäische Kommission: Erweiterungsstrategie und wichtigste Herausforderungen für den Zeitraum 20062007, S. 18.

7 Europäisches Parlament: Bericht über die Mitteilung der Kommission zur Erweiterungsstrategie und zu den wichtigsten Herausforderungen für den Zeitraum 2006-2007, (2006/2252(INI)), Ausschuss für auswärtige Angelegenheiten, 29.11.2006.

8 Europäisches Parlament: Bericht über die institutionellen Aspekte der Fähigkeit der Europäischen Union zur Aufnahme neuer Mitgliedstaaten, (2006/2226(INI)), Ausschuss für konstitutionelle Fragen, 16.11.2006.

9 Europäischer Rat: Schlussfolgerungen des Vorsitzes, 14./15. Dezember 2006.

10 Barbara Lippert: Glanzloser Arbeitserfolg von epochaler Bedeutung: eine Bilanz der EU-Erweiterungspolitik 1989-2004. in: Barbara Lippert (Hrsg.): Bilanz und Folgeprobleme der EU-Erweiterung, Baden-Baden 2004, S. 13-71, hier S. 61. 
Zweitens gibt der Gipfelbeschluss keine überzeugende Antwort darauf, wie das Problem der Kommunikation und des fehlenden politischen Führungswillens der mitgliedsstaatlichen Regierungen gelöst werden kann. Zwar stellen die Ratsschlussfolgerungen durch die Betonung des Kommunikationsaspekts eine scheinbare Abkehr der bisherigen Erweiterungsstrategie dar, in der weiter reichende Grundsatzdebatten vermieden wurden, in dem politische Konflikte „klein gearbeitet“ und ,entdramatisiert“ wurden. ${ }^{11}$ Doch wird nicht ersichtlich, über welche Mechanismen sich die Anreize für nationale Entscheidungsträger (die den nach wie vor dicksten Strang der Legitimationskette zwischen Bürgern und EU darstellen) so ändern sollten, dass diese in allen EU-Ländern eine Pro-Erweiterungs-Meinungsführerschaft übernehmen. Dieses Problem wird dadurch verschärft, dass die Faktoren, die für die Zustimmung zur Osterweiterung gesorgt haben, mit jedem Jahr Abstand zum Ende des Ost-WestKonflikts an Einfluss auf die Meinungsbildung verlieren. Das politisch-moralische Motiv, die mittel- und osteuropäischen Staaten in ein ,weltanschauliches Gegensystem ‘ zu integrieren und das Gebot historischer Gerechtigkeit verlieren von Jahr zu Jahr an Bedeutung. Das stabilitätspolitische Paradigma, das sich auf dem Gipfel von Helsinki 1999 in den Vordergrund drängte, lebte seinerseits von den Eindrücken der Balkankriege, und machte auf diese Art und Weise politischen Widerspruch von eigentlich erweiterungsskeptischen Regierungen schwierig. ${ }^{12}$ Beide Räsonnements sind heute im öffentlich-politischen Diskurs weit weniger präsent. Auch wenn Stabilitätsrisiken in den EU-Nachbarstaaten argumentierbar nach wie vor vorhanden sind und die EU gegenüber östlichen Nachbarstaaten aufgrund der gefestigten Demokratie und Rechtstaatlichkeit nach wie vor eine systemische Alternative darstellt, dürften diese Argumente heute keine ähnlich stark konsensbildende Wirkung mehr haben, wie dies im Vorfeld der Osterweiterung noch der Fall war.

Drittens stellen die Ratsbeschlüsse keine Antwort auf das Problem demokratisch legitimer Ratifizierungsmechanismen dar. Eine direkte Legitimation durch Referenden, in denen sich die vom Rat als notwendig eingeforderte Zustimmung abbilden könnte, und die zweifellos im Vergleich zur Osterweiterung sehr viel größere Kommunikationsanforderungen stellen würde, wird nicht als Option angesprochen. Ebenso wenig wurde das Problem thematisiert, dass durch die voraussichtlichen Referenden in Frankreich und anderen Staaten, ähnlich wie im Falle des Verfassungsvertrags, das Nein in einem nationalen Referendum den Fortgang eines Projektes für alle EU-Staaten lähmen würde. Diese im Ratifikationsverfahren begründete Asymmetrie könnte nur dadurch aufgebrochen werden, dass ein EU-weit einheitlicher Ratifizierungsmechanismus eingeführt wird und Regeln für den Fall gefunden werden, dass eine Minderheit den Fortgang eines Projektes (etwa eines Erweiterungsvorhabens) nicht unterstützt.

\section{Vetospieler innerhalb der EU}

Neu ist an der jüngsten Debatte um die Erweiterungsfähigkeit nicht die Sorge darum, dass die Erhöhung der Mitgliederzahl weitere Vertiefungsschritte erschweren oder gar den Status quo der Integration in Frage stellen könnte. Neu jedoch ist der ausgeprägte Wunsch zumindest einiger Regierungen und auch Europaparlamentarier, gegenüber den Bürgerinnen und Bürgern deutlich zu signalisieren, dass eine ,politische Kontrolle' über künftige Erweiterungsrunden ausgeübt werden kann und hier kein ,kommissionsgetriebener Automatis-

11 Lippert: Glanzloser Arbeitserfolg von epochaler Bedeutung, S. 60.

12 Für einen Überblick über die Erweiterungsmotive im Vorfeld der 2004/2007-Runden, siehe Lippert: Glanzloser Arbeitserfolg von epochaler Bedeutung, S. 21-23. 
mus ' herrscht. Dies ist eine Reaktion auf die oftmals als ,Erweiterungsmüdigkeit' beschriebene Skepsis, die sich in der Bevölkerung einiger Mitgliedstaaten breit gemacht hat.

\section{Nationale Referenden und parlamentarische Ratifikation}

Diese Erweiterungsskepsis erfasst die Bevölkerungen unterschiedlicher EU-Länder nicht gleichermaßen, so dass heute die Mehrheit der Bürgerinnen und Bürger in der EU-27 nicht pauschal als erweiterungskritisch einzuschätzen ist. ${ }^{13}$ Dennoch überwiegen in den Ländern der ,alten' EU-15 mittlerweile die Erweiterungsgegner (47 Prozent versus 41 Prozent), in mehreren Mitgliedstaaten hat die Erweiterungsaversion mittlerweile beachtliche Ausmaße angenommen: In Deutschland sind im Herbst 2006 fast zwei Drittel der Befragten gegen künftige Erweiterungen, in Frankreich und in Österreich teilen 58 Prozent beziehungsweise 57 Prozent diese Auffassung. Auch im traditionell erweiterungsfreundlichen Großbritannien erhielt das Lager der Beitrittsskeptiker im Laufe des Jahres 2006 immens Verstärkung (nunmehr sind 51 Prozent gegen künftige Erweiterungsrunden und nur 36 Prozent dafür; im Frühjahr hatten diese Werte noch bei 42 Prozent beziehungsweise 44 Prozent gelegen). ${ }^{14}$

Zudem hat die Ablehnung politisch eine qualitativ neue Bedeutung erlangt: Mindestens zwei EU-Länder könnten durch die Skepsis der Bevölkerung zu Vetospielern im Erweiterungsprozess werden. Dies sind Frankreich, wo seit einer Verfassungsänderung vom 1. März 2005 verbindliche Referenden für alle Erweiterungen der EU nach Kroatien vorgeschrieben sind, ${ }^{15}$ und Österreich, dessen Regierung - ohne verfassungsrechtlichen Zwang ein Referendum über einen eventuellen Beitritt der Türkei ${ }^{16}$ angekündigt hat. Sagt die Bevölkerung nur eines dieser beiden Länder ,Nein“ zum Beitrittsvertrag eines Kandidatenlandes, kann die EU nach gegebenem Vertragsstand mit diesem Vorhaben nicht weiter voranschreiten. Überdies kann nicht ausgeschlossen werden, dass mit Näherrücken einer türkischen Aufnahme andere Mitgliedstaaten ebenfalls Volksabstimmungen ankündigen. Und auch dort, wo ,lediglich' ein Plazet der Legislative erforderlich ist, kann deren Zustimmung keineswegs als selbstverständlich angesehen werden. Es ist durchaus denkbar, dass nicht alle nationalen Parlamente etwa einen Beitritt der Türkei gutheißen werden.

\section{Wachsende Skepsis im Europaparlament}

Hinzu kommt, dass seit der Einheitlichen Europäischen Akte von 1986 die Zustimmung des Europäischen Parlamentes mit absoluter Mehrheit aller Abgeordneten für den Beitritt weiterer Staaten in die Gemeinschaft unabdinglich ist. Diese Zustimmung kann das Parlament verwehren, beispielsweise wenn die EU aus seiner Sicht nicht erweiterungsfähig ist. Bereits vor der endgültigen Abstimmung ist das Europäische Parlament wesentlich in den politischen Prozess einbezogen: Die Kommission als Verhandlungsführerin berichtet dem Parlament regelmäßig über den Stand der Verhandlungen mit den Ländern, die der Union beitreten wollen. Schließlich hat das Parlament die Möglichkeit, die vor dem Beitritt

13 Eurobarometer: Die öffentliche Meinung in der Europäischen Union. Erste Ergebnisse; Befragung: September - Oktober 2006; Veröffentlichung: Dezember 2006.

14 Möglicherweise spielte hier die intensivierte Diskussion über den Zustrom von Arbeitsmigranten aus Polen und anderen neuen Mitgliedsländern eine Rolle.

15 Seit der Verfassungsänderung vom 01.03.2005 muss jeder Beitrittsvertrag der französischen Bevölkerung in einem Referendum zur Ratifizierung vorgelegt werden. Dies gilt für alle Staaten, für deren Aufnahme eine Regierungskonferenz nach dem 01.07.2005 einberufen wurde. Damit sind Rumänien, Bulgarien und Kroatien von den Referenden ausgenommen, denn die Regierungskonferenzen zur Aufnahme dieser drei Länder traten vor dem 01.07.2005 zusammen.

16 Vgl. Regierungsprogramm für die XXIII. Gesetzgebungsperiode, abrufbar unter: www.bundeskanzleramt.at/ DocView.axd?CobId=19542 (letzter Zugriff: 20.03.2006). 
weiterer Mitgliedstaaten nötigen Vertragsänderungen zur Zusammensetzung der EU-Organe und -Institutionen sowie zu den Abstimmungsregeln im Ministerrat, die im Rahmen ,kleiner Vertragsänderungen' ausgehandelt werden, abzulehnen. Anders als bei den Ergebnissen ,echter' Regierungskonferenzen zur Vertragsänderung muss das Parlament der Veränderung dieser Regeln im Rahmen des Beitritts eines Landes zustimmen. Damit ist das Parlament ein wichtiger potenzieller Vetospieler, der die Ratifizierung der Beitrittsverträge am Ende verhindern kann, und im Laufe des Prozesses durch eine öffentliche Begleitung der Verhandlungen die öffentliche Meinung gegen einen Beitritt beeinflussen kann.

Bisherige Stellungnahmen aus dem Europaparlament zur Erweiterungsstrategie nach den Aufnahmerunden 2004 und 2007 weisen eine deutlich erweiterungskritische Haltung auf, die eine Sequenzierung von Vertiefung und Erweiterung einfordert. Somit ist es kein aus der Luft gegriffenes Szenario, dass Erweiterungsgegner aus Mitgliedstaaten und Europaparlament die Ratifizierung von Beitrittsverträgen in Zukunft verhindern.

\section{Der Zusammenhang von interner Reform und Erweiterung}

Enger als je zuvor in der 50-jährigen Geschichte der europäischen Integration hängen Vertiefung und Erweiterung der Gemeinschaft derzeit unmittelbar zusammen. Dies zeigt sich beispielsweise am ,Auftrag', den der Vertrag von Nizza dem Europäischen Rat gibt: Bei 27 und mehr Mitgliedstaaten muss eine Regelung gefunden werden, wie die Kommission mit einer Anzahl von Kommissaren besetzt werden kann, die unter der Zahl der Mitgliedstaaten liegt. Darüber hinaus besteht de jure keine Notwendigkeit, das heutige, auf dem Nizza-Vertrag basierende Institutionengefüge der EU zu reformieren, um weitere Mitglieder aufzunehmen.

Politisch bestehen aber zweifellos sehr klare Zusammenhänge. So leiteten der Nizza-Vertrag ${ }^{17}$ und die folgende Erklärung des Europäischen Rats von Laeken vom 15. Dezember 2001 den so genannten ,Post-Nizza-Prozess‘ ein, der zur Einsetzung des Verfassungskonvents führte. Auch hier besteht kein juristisches Junktim ,Erweiterung erst nach Vertiefung ‘. Doch sind die politischen Einschätzungen zur Reformnotwendigkeit, die sich im Protokoll zur Zukunft der Union und der Erklärung von Laeken niederschlagen, vor dem doppelten Hintergrund der als Misserfolg betrachteten Regierungskonferenz zum Nizza-Vertrag und der in Aussicht stehenden Erweiterung um die mittel- und osteuropäischen Kandidatenländer zu sehen.

Die politischen Zusammenhänge, die sich in jüngster Zeit in den Diskussionen um Verfassungsvertrag und EU-Erweiterung aufgetan haben, sind von aktueller Bedeutung für die Diskussion um die Aufnahmefähigkeit der EU. Die Behauptung, der Verfassungsvertrag sei in Frankreich abgelehnt worden, um künftige Beitrittsrunden zu verhindern, ist zwar in dieser Vereinfachung nicht haltbar, dennoch gilt: In einigen Ländern werden institutionelle Reformen unter anderem deshalb skeptisch gesehen, weil sie Erweiterungen geradezu automatisch zu ermöglichen scheinen. So erklärt sich, dass rechte Verfassungsgegner für ein ,Nein “ gegen den Verfassungsvertrag mit dem Argument geworben haben, es verhindere den Türkeibeitritt, auch wenn diese Aussage de jure nicht haltbar ist.

17 Siehe Vertrag von Nizza, Von der Konferenz angenommene Erklärungen, 23. Erklärung zur Zukunft der Union, 2001/C 80/01. 


\section{Gründe für die gewachsene Skepsis}

Die Ablehnung weiterer Beitrittsrunden in einigen EU-Ländern hat vielfältige Gründe. Sozio-ökonomische Spannungen aufgrund hoher Arbeitslosigkeit, internationalem Wettbewerbsdruck und Standortwettbewerb sind hierfür die offensichtlichsten. Hinzu kommt die Sorge um den Einfluss des eigenen Landes in der EU sowie die Ablehnung einer kulturell, religiös, sozial und wirtschaftlich zu heterogenen EU, die unüberschaubar und unkontrollierbar erscheint. Schließlich muss auf die in vielen Ländern nach wie vor, unverdaute "EU-Erweiterung des Jahres 2004 um zehn größtenteils mittel- und osteuropäische Länder verwiesen werden. Dieser Schritt, für den in einigen EU-Ländern kein tragfähiger öffentlicher Konsens geschaffen wurde, schmälert heute die Bereitschaft zu weiteren Erweiterungen und auch zur Vertiefung - der Gemeinschaft.

Gleichzeitig hat die Zugkraft der oben angesprochenen politisch-normativen Motive, die die Erweiterungen 2004/2007 maßgeblich vorangetrieben haben, deutlich nachgelassen. Wenn damals noch die Integration der ehemaligen Ostblockstaaten in die Gemeinschaft der westlichen Demokratien als moralisch-politischer Anspruch formuliert wurde, ist heute etwa im Falle der Türkei eine wahrgenommene „Inkompatibilität mit dem jeweiligen Verständnis der normativen Grundlagen der Union und den integrationspolitischen Leitbildern" 18 Grund für die wachsende Skepsis. In Anbetracht der starken Bedeutung normativer Faktoren im Erweiterungsdiskurs der letzten Jahre ist es nicht ausgeschlossen, dass dies ein ausschlaggebender Faktor für ein mögliches Scheitern des Beitrittsprozesses (und dies kann von beiden Seiten, EU und Türkei, ausgelöst sein) wird.

\section{Der institutionelle Konsens in Auflösung}

Während es also der Erweiterungsdiskussion innerhalb der EU an positiver Zugkraft fehlt, ist die Diskussion um die Reformen des Verfassungsvertrags zerfasert. Der Konventskonsens, der alle EU-Regierungen zur Unterschrift unter das Vertragswerk gebracht hat, befindet sich in Auflösung ${ }^{19}$. Unter diesen Bedingungen einen neuen Reformkompromiss der mittlerweile 27 Mitgliedstaaten zu erzielen ist eine politisch sehr schwierige Aufgabe.

Die Reform des Institutionengefüges - über den Verfassungsvertrag hinaus - etabliert sich aber immer mehr als politische Notwendigkeit, um weitere Erweiterungen durchzuführen. Eine ungebremst fortgeführte Erweiterungsstrategie, die dem ,inneren' Zustand der Union verhältnismäßig geringe Aufmerksamkeit schenkt, führt hingegen dazu, dass die innere Reformfähigkeit der EU an den Implikationen einer extensiven Erweiterungslogik scheitert.

Die ,mechanistische“ Hoffnung, dass groß dimensionierte Erweiterungsschritte soviel Reformdruck ausüben, dass im Vorfeld eine tragfähige Lösung für eine reibungslos funktionsfähige Gemeinschaft gefunden wird, hat sich nicht bewahrheitet. Ebenso unwahrscheinlich scheint eine ,nachholende" Vertiefung zu sein, da mit zunehmender Mitgliederzahl möglicherweise eine kritische Masse für einen solchen Schritt überschritten ist. In diesem komplexen Kontext sind die Bemühungen auf EU-Ebene zu sehen, eine neue Erweiterungsstrategie zu entwickeln. Nachdem sich das Europäische Parlament bereits im März 2006 dafür ausgesprochen hatte, erteilte der Europäische Rat im Juni 2006 insbesondere auf Drängen Frankreichs und Österreichs schließlich der EU-Kommission den Auftrag, einen Bericht über die Aufnahmefähigkeit aufzusetzen.

18 Lippert: Glanzloser Arbeitserfolg von epochaler Bedeutung, S. 59.

19 Andreas Maurer/Daniela Schwarzer: The German Presidency and the EU's Constitutional Malaise, in: The International Spectator 1/2007, S. 63-79. 


\section{Für eine Sequenzierung: Konsolidierung und Konvergenz vor Erweiterung}

Für die Strategie, die Vertiefung und die Erweiterung parallel voran zu treiben, lassen sich unter geostrategischen Gesichtspunkten und im Sinne einer Stabilisierungspolitik in EU-angrenzenden Ländern gute Argumente finden. Allerdings ist diese Doppelstrategie nur dann sinnvoll, wenn sie in beiden Bereichen, Vertiefung und Erweiterung, Erfolg verspricht. Wird die EU indes, und dies zeigt die Realität der letzten Jahre, zunehmend fragmentiert und mit der Erarbeitung ihrer inneren Handlungsgrundlagen befasst, so läuft sie Gefahr, zu einer kraftlosen Modernisierungsmacht zu werden. Natürlich ist die Aufnahme neuer Mitglieder nicht die alleinige Ursache aller akuten Probleme der europäischen Integration. In zahlreichen Bereichen hat sich die Erweiterung unspektakulär ausgewirkt. Es gibt nach der Erweiterung 2004 Hinweise auf ein höheres Maß an ,Konsenskultur', und es sind keineswegs vornehmlich die Neumitglieder, die als ,Störenfriede“ auffallen, sondern in der Tendenz die größeren Mitgliedstaaten. Doch sind die Entscheidungsprozesse in einigen Politikfeldern angesichts von 27 Mitgliedern deutlich komplexer geworden, Kommunikationsprozesse sind weniger direkt und schlagen deshalb des Öfteren fehl. In einigen sensitiven Politikfeldern (etwa Justiz und Inneres) kam es bereits jetzt zu einer Abnahme des legislativen Outputs. ${ }^{20}$

Die Kommission hat dieses und andere Probleme in ihrem Bericht zweifellos angesprochen. Allerdings hat sie nicht nur, wie oben erwähnt, ein eher weiches Bedingungsverhältnis zwischen interner Festigung und Erweiterung konstruiert, sondern blieb auch hinter Forderungen aus dem Europäischen Parlament zurück. Sowohl der Stubb-Bericht als auch der Brok-Bericht hatten ja mit wesentlich detaillierteren Postulaten ein restriktiveres Vorgehen angemahnt.

Um die Erweiterungsfähigkeit der Europäischen Union überhaupt gewährleisten zu können sollten daher klare Leitlinien definiert werden, anhand derer die Aufnahmefähigkeit der EU in Bezug auf konkrete Beitrittsaspiranten bewertet wird. Hierbei geht es nicht um die dauerhafte Definition geografischer Grenzen der EU, sondern um die dynamische Festlegung funktionaler Grenzen der Integration. Bei einer differenzierten Anwendung der Kriterien besteht die Möglichkeit, dass die EU als aufnahmefähig für ein Land, nicht aber für ein anderes bewertet wird.

Das zunehmende wirtschaftliche Gefälle in der wachsenden Union ist in zentraler Weise verantwortlich für sozio-ökonomische Spannungen, finanzielle Belastungen und eine sinkende Akzeptanz der Erweiterung in einigen Mitgliedsländern. Ein Großteil der realen, potenziellen oder vermeintlichen Negativwirkungen der Erweiterung besteht aus Sicht zahlreicher Mitgliedsländer und nationaler Öffentlichkeiten darin, dass Beitritte kostenträchtig sind und dass Wohlfahrts- und Lohndifferenziale Migrations- und Delokalisierungsbewegungen auslösen. Manche dieser Befürchtungen sind überbewertet, gar virtuell, andere wiederum äußerst konkret und empirisch belegbar.

Die bisherige Erweiterungslogik basiert darauf, das Wohlfahrtsgefälle zwischen ,Alt und Neu-EU’ zu überwinden, indem Kohäsion und Konvergenz durch beziehungsweise nach Inklusion geschaffen werden. Mit Blick auf die Integrationsfähigkeit der Union wäre es besser, bereits vor einem Beitritt ein Mindestmaß an Konvergenz herzustellen. Dies würde sicherlich schwerer sein als ohne Mitgliedschaft, doch ginge es ja lediglich um eine relative Annäherung. Für die Erweiterungspolitik der Union hieße dies, dass die EU eine Mindestschwelle oder einen Korridor ökonomischer Konvergenz als notwendige Voraussetzung für

20 Sara Hagemann/Julia De Clerck-Sachsse: Decision-making in the Enlarged Council of Ministers: Evaluating the Facts, CEPS Policy Brief 119, Januar 2007. 
die Verleihung des Kandidatenstatus festlegen könnte. Immerhin „spricht [...] viel dafür, dass bei zu großem Abstand der Wirtschaftsleistung (zum Beispiel gemessen am BIP pro Kopf) Konvergenz erheblich erschwert wird, wenn nicht gar in Divergenz umschlägt, was insbesondere für die beitretenden Länder, wie auch für die politische Stabilität des Gesamtgebildes verheerende Folgen hätte“" ${ }^{21}$

Ökonomische Minimalkonvergenz würde zahlreichen , sensiblen` Erweiterungsfolgeproblemen die Spitze nehmen. EU-interner Wanderungsdruck würde reduziert, kostenintensive Aspekte des Beitritts würden abgemildert (Regional- und Strukturpolitik, aber auch Gemeinsame Agrarpolitik, da ein geringeres ökonomisches Entwicklungsniveau in der Regel auch mit der Existenz eines großen Landwirtschaftssektors einhergeht). Würden lediglich Kandidaten mit dem Gütesiegel eines soliden wirtschaftlichen Konvergenzgrads in die Union aufgenommen, so wäre auch für spätere Euro-Zonen-Erweiterungen das Risiko reduziert, dass nominal regelkonforme Aspiranten aufgrund fehlender realwirtschaftlicher Grundlagen zu unsicheren Kantonisten werden.

Kategorien wie soziokulturelle Diversität oder die Furcht vor Einflussverlust sind objektiv schwer ,abschätzbar', und (wie etwa das Kriterium der Identität) als normative Setzungen ohnehin nicht durch ein technisches Raster der Folgewirkungsbeurteilung erfassbar. Allerdings sind es bedeutsame Faktoren, um öffentlichen Konsens für weitere Erweiterungsschritte zu schaffen. Regelmäßige und (nach Kriterien und Ländern detaillierte) Umfragen können begleitend zum gesamten Beitrittsprozess als ,Gradmesser' für diese sensiblen Themen dienen. Je klarer die Akzeptanzprobleme offen gelegt werden, desto größer ist die Chance für Politiker in der EU und in den Kandidatenländern, hierauf zu reagieren und die öffentliche Meinung in ihrem Sinne zu beeinflussen. Geschieht dies nicht, kann über eine Stärkung der Ratifikationsmechanismen stärker als zuvor gesichert werden, dass keine Erweiterungsschritte beschlossen werden, die die EU-Bevölkerung von der EU, entfremden '.

Auch wenn immer wieder Studien versuchen zu belegen, dass das EU-Institutionengefüge nach Erweiterung und ohne Verfassungsvertrag reibungslos funktioniert, muss anerkannt werden, dass die Entscheidungsmechanismen auf die Herausforderung, 27 Länder zu gemeinsamen Beschlüssen zu bewegen, mit einem neuen Modus Vivendi reagiert hat, dessen Konsequenzen für eine EU-35 oder EU-40 zu überdenken sind. Kleinere Ländergruppen, die gemeinsam jenseits der EU-Institutionen voranpreschen oder intergouvernementale Absprachen der größten Staaten im Vorfeld von Ratsverhandlungen auf Kosten der kleineren Staaten, sind einerseits pragmatische Lösungen, um die Entscheidungsfähigkeit aufrecht $\mathrm{zu}$ erhalten. Andererseits ist bei einer weiter wachsenden Gemeinschaft ernsthaft die Frage zu stellen, in wie weit die Bürgerinnen und Bürger und die nationalen Institutionen einer nötigen Ausweitung der Supranationalität zustimmen würden, um die Kohärenz und Handlungsfähigkeit der Gemeinschaft dauerhaft zu festigen. Künftige Erweiterungen, die ohne über den Verfassungsvertrag hinausgehende Reformen durchgeführt würden, sind gefährlich, weil sie tendenziell die Gemeinschaftsinstitutionen schwächen und machtbasierte Asymmetrien zwischen den Mitgliedstaaten fördern.

Parlamente frühzeitig in das Erweiterungsgeschehen einzubeziehen kann dazu beitragen, die Akzeptanz von weiteren Beitritten und ihren Konsequenzen zu ermitteln und die Zustimmung zu Beitritten in den Öffentlichkeiten der Mitgliedstaaten zu verbessern. Der im BrokBericht enthaltene Vorschlag, das Europäische Parlament an der Entscheidung über die Er-

21 Hermann Clement (unter Mitarbeit von Volkhart Vincentz): Die neuen Nachbarn und die Europäische Union Möglichkeiten und Grenzen der Integration am Beispiel Ukraine und Belarus, Osteuropa-Institut München, Working Paper Nr. 256, Januar 2005, S. 103. 
öffnung von Beitrittsverhandlungen zu beteiligen, sollte aufgegriffen werden. Überdies sollten die nationalen Parlamente in Form eines konsultativen Votums vor einem Ratsbeschluss zur Aufnahme von Verhandlungen eingebunden werden.

Ein wichtiges Gebot einer derart verstandenen Politik wäre es, die Beitrittsprozesse etwa der Türkei und der Balkanstaaten in der öffentlichen Wahrnehmung zu entkoppeln. Sollte es sich als politisch unrealistisch erweisen, dass der Türkeibeitritt in der EU ratifiziert wird oder dass die Türkei ausreichende Modernisierungsschritte unternimmt, um die Kriterien zur Mitgliedschaft zu erfüllen, rückt die Frage nach Alternativen zur Vollmitgliedschaft auf die politische Agenda. Eine stringente und ehrliche Prüfung der Aufnahmefähigkeit der EU kann diesen Erkenntnisprozess beschleunigen, und ein langsames, möglicherweise bewusst ignoriertes und daher politisch nicht aktiv begleitetes Auseinanderdriften von EU und Kandidatenländern verhindern.

Bei alledem gibt es keine Gewähr dafür, dass eine auch noch so konsistente Erweiterungsvorbereitung die Aufnahmebereitschaft aller beziehungsweise ,kritischer' nationaler Öffentlichkeiten sichert. Selbst die möglichst präzise Skizzierung beziehungsweise Reduktion von ökonomischen, finanziellen oder strategischen Problemeffekten konkreter Beitritte kann die Zustimmung zur Aufnahme neuer Mitglieder nicht garantieren. Gleichwohl sind alle Anstrengungen zur Stärkung der Legitimationsbasis von Erweiterungen wünschenswert und unumgänglich. Wünschenswert, weil sie allgemeineren Tendenzen zum Loyalitätsentzug der Bürger gegenüber der EU entgegenwirken, unumgänglich, weil sonst das Risiko von erweiterungspolitischen ,Betriebsunfällen', also etwa einem ,Nein“ in einem nationalen Referendum zu einem Beitrittsvertrag, zunimmt.

\section{Ein respektvoller Umgang mit den Kandidatenländern}

Politische Äußerungen innerhalb der EU, die als Ablehnung oder Skepsis gegenüber dem Beitritt eines Mitgliedslandes interpretiert werden können, können politisch destabilisierende Effekte in den Beitrittsländern haben. Wenn also die Zügelung des Erweiterungsablaufs zu einer Verlangsamung der Verhandlungen führen sollte, muss dieser Prozess in einen politischkonzeptionellen Rahmen eingefügt werden, der die Umgestaltungs- und Konsolidierungsdynamik des Kooperationsgeschehens nicht entkräftet. Denn eine Neuausrichtung der Erweiterungspolitik soll und darf nicht dazu führen, dass die EU an Transformationskraft im Umgang mit Drittstaaten einbüßt. Ganz im Gegenteil geht es darum, diese Transformationskraft abzusichern, indem sie auf ein solides inneres Fundament gestellt wird. In der Tat muss hierbei auch die von Erweiterungskommissar Rehn geforderte Zusammenführung eines eher stabilitätsorientierten und eines stärker auf Fragen der Handlungsfähigkeit konzentrierten Diskurses erfolgen.22

Der zentrale Punkt bei der Formulierung einer neuen Erweiterungspolitik mit Augenmaß ist, die Diskussion um Aufnahme- und Expansionsfähigkeit als konstruktiven Bestandteil der Diskussion um die Zukunft der europäischen Integration zu führen. Integrationsfähigkeit und eine praktisch-politische Ausbuchstabierung einschlägiger Kriterien sollten nicht als Ausdruck einer unreflektierten Generalablehnung gegen neue Mitgliedschaften missverstanden, sondern als notwendige Voraussetzung für den Fortgang des Erweiterungsgeschehens kommuniziert werden. Als solche liegt die Diskussion und die Verankerung konkreter Fähigkeitsmerkmale auch im Eigeninteresse von Mitgliedschaftsbewerbern. Ein dem Grundsatz innerer Konsolidierung und Funktionsfähigkeit verschriebener Umgang mit beitrittswilligen Partnern ist also nicht Erweiterungsverhinderungspolitik, sondern Erweiterungsermöglichungspolitik.

22 Olli Rehn: Debate on Enlargement in the EP, Straßburg, 13.12.2006, Speech/06/797. 
Gleichzeitig darf die Union bei der Diskussion um die Herstellung von Integrations- und somit Erweiterungsfähigkeit auch nicht die Augen vor dem Thema Nichtmitgliedschaft verschließen. Nicht nur dann, wenn sich in den kommenden Jahren abzeichnen würde, dass eine Beitrittsperspektive etwa der Türkei aufgrund der politischen Konstellationen und der öffentlichen Meinung nicht nur in der EU, sondern auch im Beitrittsland, unrealistisch erscheint, muss proaktiv eine Diskussion über Alternativen zur Vollmitgliedschaft für fehlgeschlagene Unionsanwärter geführt werden. Allein schon die Vorgabe, ergebnisoffener ' Beitrittsverhandlungen legt die frühzeitige Skizzierung von Auffangoptionen nahe. Hierbei könnten die Erfahrungen aus dem Bereich der Europäischen Nachbarschaftspolitik, insbesondere die Etablierung von vertraglichen Kooperationsbeziehungen „neuen Typs“ mit den ENP-Partnern, für die substanzielle, institutionelle und rechtliche Ausgestaltung solch begrenzter Andockabkommen nützlich sein. ${ }^{23}$

Mit dieser Strategie hätte die EU die größten Chancen, ihre strategische Sicherheit zu gewährleisten. Immer wieder wird die Erweiterungspolitik als einzig gangbarer Weg dargestellt, etwa in Bezug auf die Türkei, die Ukraine oder den Balkan eine wirksame Stabilisierungspolitik zu machen. Angesichts der Gefahr einer Nichtratifizierung von Beitrittsverträgen oder eines Abbruchs der Verhandlungen ist diese Strategie heute nicht mehr in jedem Falle glaubhaft, und muss durch ein ergebnisoffeneres Vorgehen mit gleichem Ziel (Stabilisierung der Länder, enge strategische Anbindung an die EU) fortgeführt werden.

\section{Implementierungschancen}

Der schwache Kompromiss des Europäischen Rats von Dezember 2006, der in der Substanz wenig über die bereits in den vergangenen Jahren erfolgte Entwicklung hinausgeht, zeigt: Es gibt heute noch keine starke Mehrheit für eine Sequenzierung von Vertiefung und Erweiterung der Europäischen Union. Hierüber kann auch nicht hinwegtäuschen, dass in der Union gegenwärtig faktisch Einigkeit darüber herrscht, dass ohne eine Überwindung der Blockade des Verfassungsvertrags keine neuerliche Erweiterung durchsetzbar ist. Ein strapazierfähiger Erweiterungskonsens wurde jedenfalls zum gegenwärtigen Zeitpunkt noch nicht etabliert.

Jenseits der fragilen Harmonie des neuen Erweiterungskonzepts zeichnen sich hingegen deutlich zwei Lager ab. Zu den ,Sequenzierern“ gehören Frankreich, Österreich (allerdings nicht mit Blick auf den Westbalkan), die Niederlande sowie sehr eindeutig das Europäische Parlament. Auch Deutschland ist zu dieser Gruppe zu zählen, zumindest hat Bundeskanzlerin Angela Merkel in einer Rede vor Beginn der deutschen EU-Ratspräsidentschaft bezweifelt, dass beide Prozesse parallel zu bewältigen sind. Zur Gruppe derer, die die Erweiterung rasch vorantreiben wollen, gehören aus unterschiedlichen Motiven heraus Großbritannien, Italien und die meisten der seit 2004 beigetretenen Mitgliedstaaten.

Sogar für den Fall, dass die politische Substanz des Verfassungsvertrags in den kommenden Jahren implementiert wird, muss bezweifelt werden, dass dies reicht, um die sich momentan abzeichnenden Kandidaturen, also Westbalkan und vor allem Türkei, in ihrer Gänze zu bewältigen. Insofern greift auch die Einschätzung des Konstitutionellen Ausschusses des Europäischen Parlaments zu kurz. Dieser hatte in seinem Bericht konstatiert, dass der Verfassungsvertrag ,bereits Lösungen für die meisten Reformen der EU enthält, die notwendig sind, damit die Union ihre Erweiterungszusagen erfüllen kann“.24

23 Vgl. Barbara Lippert: Assoziierung plus gesamteuropäische Aufgabenkonföderation: Plädoyer für eine selbstbewusste EU-Nachbarschaftspolitik, in: integration 2/2006, S. 149-157.

24 Europäisches Parlament: Bericht über die institutionellen Aspekte der Fähigkeit der Europäischen Union zur Aufnahme neuer Mitgliedstaaten. 